\title{
Lithographically Defined Epitaxy via Focused Ion Beam Induced Lattice Damage
}

\author{
B.D. Myers ${ }^{1,2}$, B.L. Stevens ${ }^{1}$, S.A. Barnett ${ }^{1}$ and V.P. Dravid ${ }^{1,2}$
}

1. Department of Materials Science and Engineering, Northwestern University, 2220 Campus Drive, Evanston, IL 60208

2. NUANCE Center, Northwestern University, 2220 Campus Drive, Evanston, IL 60208

Focused Ion Beam (FIB) induced damage is a significant issue in nearly every application of this technology, including preparation of TEM, EBSD and atom probe samples, ion beam lithography and ion beam induced deposition [1]. In addition, energetic ions are well known to cause lattice damage in ion implant operations common in semiconductor processing [2]. Epitaxial thin film growth represents an important class of processes for many different materials systems and technologies. These processes rely on a film/substrate system with reasonable lattice matching, an appropriate deposition method and a pristine substrate lattice. However, for many materials systems, such as functional ceramics, patterning of epitaxial layers is challenging due to difficult etching processes and high deposition temperatures. We present a novel method for lithographically defining epitaxial structures via focused ion beam induced lattice damage.

To study the effects of FIB damage on epitaxial growth, we chose to deposit zinc oxide on c-axis oriented sapphire $(\sim 16.8 \%$ lattice mismatch). C-axis oriented sapphire samples were cleaned with $\mathrm{Ar} / \mathrm{O}_{2}$ plasma and then patterns were exposed using a $\mathrm{Ga}^{+}$ion beam at different beam energies in a FEI Helios NanoLab. Zinc oxide films were deposited by DC reactive sputtering at different substrate temperatures. With $5 \mathrm{keV}$ ion beam energy, we found that the ion induced lattice damage with an area dose of $250 \mu \mathrm{C} / \mathrm{cm}^{2}$ was sufficient to inhibit epitaxial growth. The epitaxial and polycrystalline regions are clearly identified in Figure 1a via channeling contrast in the FIB image. The SAED patterns of interfaces in ion irradiated and non-irradiated regions shown in Figure $1 \mathrm{~b}$ and $1 \mathrm{c}$ confirm the disruption of epitaxial growth in the ion damaged regions. In addition, the process is capable of achieving sub-100nm feature sizes as indicated by the $8 \mathrm{keV}$ exposed patterns in Figure 2.

To optimize the FIB induced damage mechanism for this process, we investigated the balance between ion range, ion sputtering and ion induced lattice damage. The optimum ion energy is achieved when the maximum amount of damage is induced in the near surface of the substrate with the lowest sputtering yield. Figure 3 shows results from TRIM calculations of vacancy generation within the top 3 and $5 \mathrm{~nm}$ of the substrate as well as sputtering yield for different ion beam energies. These data suggest that sputtering yield decreases with decreasing ion energy, resulting in a more planar surface, low energy ions are not as efficient at generating defects. High energy ions generate a larger number of defects, but they have a larger range in the sample and therefore do not generate as many near-surface defects.

Directed epitaxial growth via FIB induced lattice damage is a novel method for control of thin film texture with nanometer-scale precision. The process efficiency is similar to methods such as electron beam lithography and is much more efficient than typical FIB lithography via surface sputtering or beam induced deposition. We demonstrate the concept with the $\mathrm{ZnO} / \mathrm{Al}_{2} \mathrm{O}_{3}$ system, but the method is likely applicable to a range of different epitaxial film/substrate systems. In 
addition, the concept could be extended to different ion sources (eg, Ar, He, Xe) and from FIB direct writing to broad beam exposure through a mask.

\section{References}

[1] C.A. Volkert and A.M. Minor, MRS Bulletin. 32:5 (2007) 389.

[2] G. Dearnaley, Annual Review of Materials Science. 4 (1974) 93.

[3] This material is based on work supported by the MRSEC program of the National Science Foundation (DMR-0520513) at the Materials Research Center of Northwestern University.

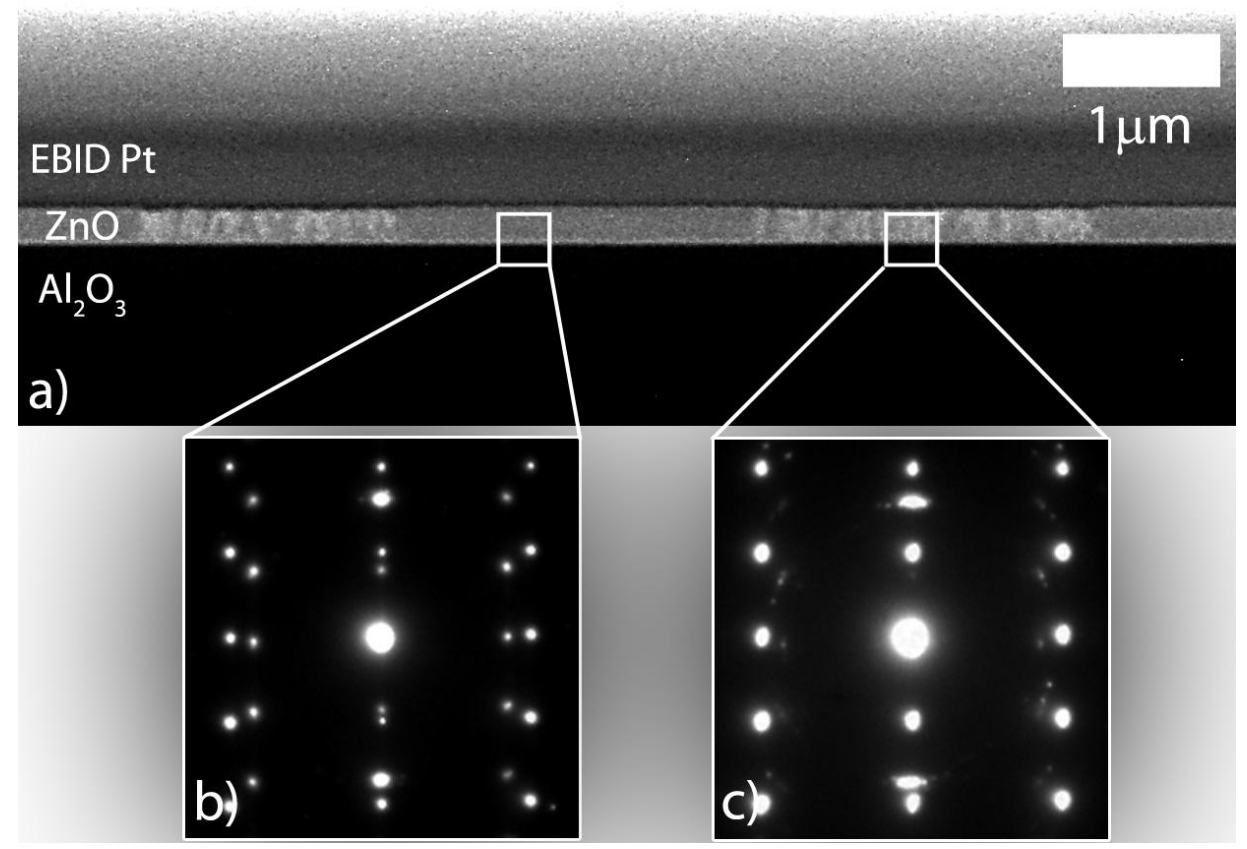

FIG. 1. a) FIB image of cross section through as-deposited $\mathrm{ZnO}$ on $\alpha-\mathrm{Al}_{2} \mathrm{O}_{3}$. SAED patterns of $\mathrm{b}$ ) epitaxial film and c) polycrystalline film on $5 \mathrm{keV}$ ion damaged region.

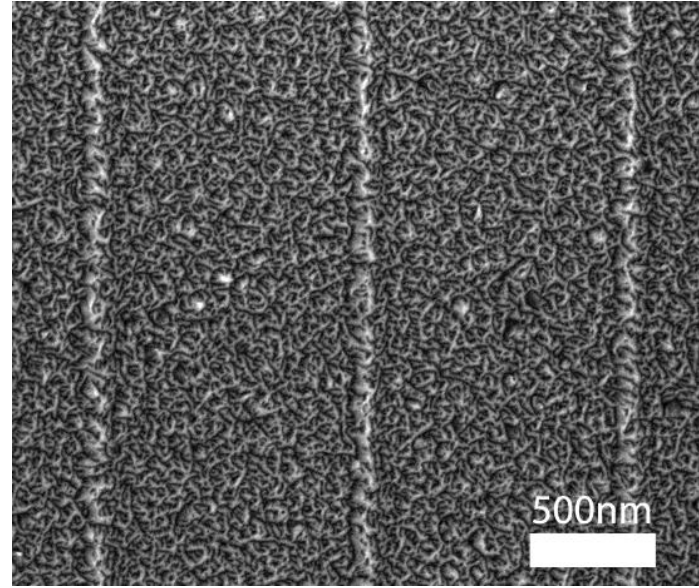

FIG. 2. SEM image of as-deposited $\mathrm{ZnO}$ film featuring sub-100nm lines patterned by $8 \mathrm{keV}$ ion damage to the substrate.

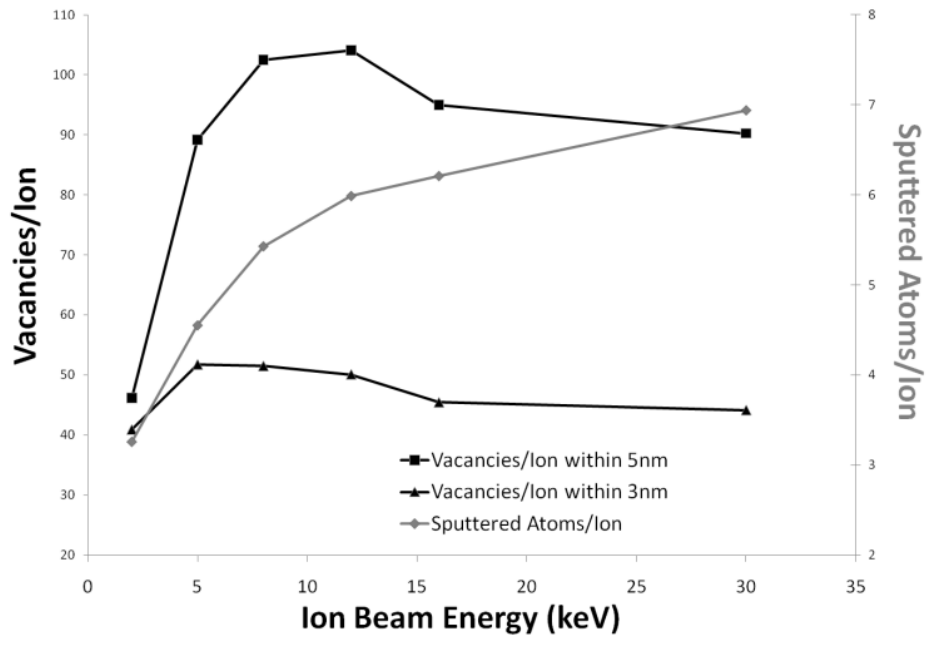

FIG. 3. Vacancy production and sputter yield dependence on beam energy from TRIM calculations of $\mathrm{Ga}^{+}$ion scattering in $\alpha-\mathrm{Al}_{2} \mathrm{O}_{3}$. 